\title{
Correlações entre compostos fenólicos e atividade antioxidante em casca e polpa de variedades de uva de mesa
}

\author{
Correlations between the phenolic compounds and antioxidant activity in the skin and \\ pulp of table grape varieties
}

\author{
Angela Vacaro de Souza ${ }^{1 *}$, Marcos Ribeiro da Silva Vieira², Fernando Ferrari Putti ${ }^{1}$ \\ ${ }^{1}$ Universidade Estadual Paulista (UNESP), Faculdade de Ciências e Engenharia, Tupã/SP - Brasil \\ 2 Universidade Federal do Pará (UFPA), Altamira/PA - Brasil
}

\section{${ }^{*}$ Corresponding Author}

Angela Vacaro de Souza, Universidade Estadual Paulista (UNESP), Faculdade de Ciências e Engenharia, Câmpus de Tupã, Rua Domingos da Costa Lopes, 780, Jardim Itaipu, CEP: 17602-496, Tupã/SP - Brasil, e-mail: angela@tupa.unesp.br

Cite as: Correlations between the phenolic compounds and antioxidant activity in the skin and pulp of table grape varieties. Braz. J. Food Technol., v. 21, e2017103, 2018.

\section{Resumo}

Uvas são consideradas excelentes fontes de compostos fenólicos, quando comparadas a outros vegetais; porém, a grande diversidade entre as cultivares resulta em frutos com diferentes características, tanto de sabor quanto de coloração, o que, certamente, está associado com o conteúdo e o perfil de compostos polifenólicos. Cascas e polpas de amostras de uva no ponto de consumo - tipos "Itália", "Brasil", "Rubi", "Thompson" e "Niágara Rosada" - da região de Campinas e Jundiaí, Estado de São Paulo, foram estudadas com o objetivo de avaliar a atividade antioxidante, a atividade enzimática da peroxidase e da polifenoloxidase e os teores de compostos fenólicos e de ácido ascórbico. A fim de verificar as correlações entre as cascas e polpas das variedades e as características, utilizou-se a Correlação de Pearson, a distância generalizada de Mahalanobis $\left(D^{2}\right)$ e, por fim, o agrupamento das diferentes respostas através da análise multivariada. Verificou-se que as cascas das uvas analisadas apresentaram correlação positiva com o conteúdo de compostos fenólicos, a atividade da enzima polifenoloxidase e com o teor de ácido ascórbico, exceto a cultivar "Niágara Rosada", a qual mostrou maiores valores para estas avaliações. Não houve correlação entre o índice de atividade antioxidante com as demais análises realizadas. As cascas se mostraram uma ótima fonte para estes compostos.

Palavras-chave: Vitis sp.; Ácido ascórbico; Índice de atividade antioxidante.

\section{Abstract}

Grapes are considered a major source of phenolic compounds when compared to other vegetables, but the great diversity amongst the cultivars results in fruits with different characteristics of taste and colour, which is certainly associated with the polyphenol content and profile. Skins and pulps of ripe samples of "Itália", "Brasil", "Rubi", "Thompson" and "Niagara Rosada" grapes from the Campinas and Jundiaí regions - SP state - Brazil, were studied with the objective of evaluating the antioxidant activity, enzymatic activities of peroxidase and polyphenoloxidase, phenolic compounds and ascorbic acid. In order to verify correlations between the skins and pulps of the varieties and the characteristics studied, Pearson's correlation, the generalized distance of Mahalanobis (D2) and finally the grouping of the different responses using the multivariate analysis, were carried out. It was verified that the skins of the grapes analysed had a positive correlation with the phenolic compounds, polyphenoloxidase activity and ascorbic acid content, except for "Niagara Rosada", showing higher levels for these evaluations. There was no correlation between the antioxidant activity and the other analyzes. This shows that for the majority of the varieties evaluated, the skins are a great source of these compounds.

Keywords: Vitis sp.; Ascorbic acid; Antioxidant activity index. 


\section{Introdução}

O homem necessita de uma alimentação sadia, rica em nutrientes, e isto pode ser alcançado com partes de alimentos que normalmente são desprezadas. Assim, é importante a utilização de cascas, talos e folhas, pois o aproveitamento integral dos alimentos, além de diminuir os gastos com alimentação e melhorar a qualidade nutricional do cardápio, reduz o desperdício e torna possível a criação de novas receitas, como, por exemplo, sucos, doces, geleias e farinhas (GONDIM et al., 2005).

Nos últimos anos, houve um interesse crescente na determinação de fontes dietéticas adequadas de compostos fenólicos antioxidantes. As uvas (Vitis sp.) estão entre os frutos consumidos em maiores quantidades em todo o mundo, sejam processados ou em sua forma natural, constituindo, este vegetal, uma das maiores fontes de compostos fenólicos (MANACH et al., 2005). Polifenóis têm sido associados ao potencial bioativo das uvas, devido às suas atividades antioxidantes, anti-inflamatórias, anticancerígenas e antibacterianas (BAGCHI et al., 2000; GRIS et al., 2011).

Os compostos fenólicos das uvas são responsáveis não só por importantes características do vinho - como cor, adstringência, sabor e corpo - mas por estarem, também, relacionados a efeitos benéficos para a saúde humana, com o consumo moderado da bebida (LÓPEZ-MIRANDA et al., 2016). Os compostos fenólicos desempenham importante papel na proteção celular, pois são capazes de sequestrar ou inibir as diversas espécies de oxigênio reativo, transferir elétrons para radicais livres, ativar enzimas antioxidantes e inibir enzimas oxidases (DUMITRIU et al., 2015), desempenhando forte ação na prevenção do estresse oxidativo, apontado como causa de algumas doenças, como arteriosclerose, diabete e doenças neurodegenerativas (ASADI et al., 2010). Ademais, esses compostos estão frequentemente associados à inibição do crescimento de células cancerígenas (JARA-PALACIOS et al., 2015; LEE et al., 2006; VU et al., 2012), além da ação anti-inflamatória, analgésica, gastroprotetora (LAJILI et al., 2016) e antimicrobiana (CETIN-KARACA; NEWMAN, 2015). Gonçalves et al. (2017) citam em seu trabalho a capacidade dos compostos fenólicos atenuarem o estresse oxidativo, relatando, ainda, a presença destes compostos em grandes quantidades em bagaço de uvas Merlot.

Assim como os compostos fenólicos, a vitamina $\mathrm{C}$ também está presente nas uvas. Esta promove proteção contra espécies reativas produzidas durante os processos de fotossíntese e de respiração, e está envolvida no crescimento celular, bem como atua como cofator de importantes enzimas na síntese de antocianidina e outros metabólitos secundários (SOARES et al., 2004).

Além de atuarem na redução da atividade antioxidante, as enzimas promovem o escurecimento enzimático das bagas, que tem sido um sério problema de conservação dos frutos. Em uvas, o escurecimento oxidativo pode ocorrer também quando a polifenoloxidase (PPO) e os fenóis entram em contato, numa situação em que as bagas tenham sofrido danos (AWAD, 1993). Há, ainda, a oxidação de fenóis resultante da atividade de peroxidases (POD) ou mesmo sem participação de enzimas, sendo que este conhecimento é fundamental para a viabilização de técnicas que reduzam as perdas decorrentes do escurecimento das bagas de uvas armazenadas. Este trabalho teve como objetivo investigar as relações entre a quantidade de compostos fenólicos, a atividade das enzimas peroxidase e polifenoloxidase, o índice de atividade antioxidante e a quantidade de ácido ascórbico de diferentes variedades de uvas, em suas partes casca e polpa.

\section{Material e métodos}

Foram utilizadas polpas de frutos maduros das variedades "Itália", "Brasil", "Rubi", "Thompson Seedless" e "Niágara Rosada" da região de Campinas, São Paulo (Latitude: S $22^{\circ} 53^{\prime} 20^{\prime \prime}$, Longitude: W 4704' 40" e Altitude de $680 \mathrm{~m})$.

O experimento foi conduzido em delineamento inteiramente casualizado (D.I.C.), composto por cinco variedades de uva em dois locais: casca e polpa. Cada tratamento foi composto de três repetições, cada uma com 10 frutos. As análises foram realizadas em triplicata.

Foi realizada análise de compostos fenólicos totais na matéria fresca, de acordo com o método espectrofotométrico Folin-Ciocalteu (SINGLETON; ROSSI JÚNIOR, 1965) e os resultados foram expressos em mg de ácido gálico $\mathrm{g}^{-1}$.

A atividade enzimática foi determinada a partir de $0,5 \mathrm{~g}$ de tecido vegetal fresco (polpa) triturado em graal de porcelana com $\mathrm{N}_{2}$, contendo $5 \mathrm{~mL}$ de tampão-fosfato de sódio 0,1 M, pH 6,0, sendo o homogenado resultante centrifugado por 10 minutos a $10.000 \mathrm{rpm}$, a $4{ }^{\circ} \mathrm{C}$. O sobrenadante obtido foi utilizado como fonte de enzimas. $A$ atividade da peroxidase foi determinada segundo o método descrito por Lima et al. (1999). A leitura foi realizada em espectrofotômetro Ultrospec 2000/Pharmacía Biotech, utilizando o comprimento de onda de $505 \mathrm{~nm}$. Uma unidade de atividade de peroxidase foi definida como o correspondente à variação de uma unidade de absorbância por minuto por $\mathrm{mL}$ de amostra.

$A$ atividade da PPO foi determinada de acordo com o método descrito por Cano et al. (1997). A leitura foi realizada em espectrofotômetro a $395 \mathrm{~nm}$. Uma unidade de atividade de PPO foi definida como o correspondente à variação de uma unidade de absorbância por minuto por $\mathrm{mL}$ de amostra.

Para a determinação do conteúdo de ácido ascórbico (mg $100 \mathrm{~g}^{-1}$ de polpa), foi utilizado o método proposto por Terada et al. (1979). A leitura foi realizada 
em espectrofotômetro a $540 \mathrm{~nm}$ e, para a determinação da curva padrão, foi utilizada solução padrão de ácido ascórbico.

A determinação da atividade antioxidante pelo método DPPH baseia-se na redução deste radical livre, relativamente estável, DPPH (2,2 difenil-1-picrilhidrazila), em solução alcoólica, o qual, na presença de antioxidantes doadores de hidrogênio, captura estes elétrons, mudando a coloração de violeta para amarela, passando para sua forma estável, DPPH-H. Para facilitar a visualização dos dados, um Índice de Atividade Antioxidante (IAA), proposto pelos pesquisadores Scherer e Godoy (2009), foi utilizado neste trabalho. Este índice é a razão entre a concentração final de DPPH de 118,296 $\mu \mathrm{g} \mathrm{mL} \mathrm{m}^{-1}$ 0,3 mM e o índice de $50 \%$ de inibição $\left(\mathrm{CE}_{50}\right)$, calculado pela regressão linear de cada uma das leituras. Os autores demonstraram que extratos de plantas com IAA menor que 0,5 foram pobres em atividade antioxidante; as plantas com IAA no intervalo de 0,5 a 1,0 foram classificadas como moderadas; as de índice 1,0 a 2,0 apresentaram forte atividade antioxidante, e as de razão superior a 2,0 foram classificadas como plantas de atividade antioxidante muito forte.

A fim de investigar as relações entre as variáveis do estudo, foi adotada a Análise de Correlação de Pearson, a qual indica a existência positiva ou negativa entre duas variáveis, sendo que foi adotado $\alpha=5 \%$ (coeficiente de correlação), para verificar a significância. As análises foram realizas pelo software Statistica e Sigmaplot.

Determinou-se a distância generalizada de Mahalanobis $\left(D^{2}\right)$ para a análise de agrupamento das cascas e polpas das cultivares de uva de mesa, pelo método da ligação média entre grupo (UPGM). Após essa análise, determinou-se o Coeficiente de Correlação Cofenética (CCC), assim possibilitando o ajuste entre a matriz de dissimilaridade e o dendrograma obtido (CRUZ et al., 2004).

Foi realizada a análise multivariada via componentes principais (ACP), a fim de verificar o agrupamento das diferentes respostas de cultivares de uva em casca e polpa, tendo como variáveis as características bioquímicas (teor de compostos fenólicos, atividade antioxidante, atividade das enzimas PPO e POD, e teor de ácido ascórbico), e assim simular como é a relação entre essas variáveis (VÍTOLO et al., 2012). Para a realização da análise, utilizou-se o software Statistica usando o critério de absorção mínima de $80 \%$ nos dois primeiros componentes principais e, assim, simular possível relação entre as variáveis (CRUZ et al., 2004).

\section{Resultados e discussão}

Correlações significativas e positivas ocorreram entre compostos fenólicos e a atividade da enzima polifenoloxidase e a quantidade de ácido ascórbico encontrada nos frutos (Tabela 1). Sabe-se que a atividade antioxidante de produtos derivados da uva é influenciada não apenas pelo seu teor total de polifenóis, mas também pela sua composição fenólica. Em geral, correlações positivas são observadas entre a atividade antioxidante determinada pelo método utilizado no presente trabalho e os compostos fenólicos (TAGLIAZUCCHI et al., 2010; BURIN et al., 2014). No entanto, alguns autores observaram que não há correlação ou há correlação negativa entre o teor de polifenois e a atividade antioxidante da PPO, assim como encontrado no presente experimento (NIXDORF; HERMOSÍN-GUTIÉRREZ, 2010).

A contribuição da vitamina C para a atividade antioxidante das frutas é altamente variável. Alguns estudos mostram que a atividade antioxidante nem sempre se correlaciona positivamente com a quantidade de vitamina $\mathrm{C}$ (ácido ascórbico) (GUO et al., 2003).

Os resultados apresentados na Figura 1 mostram que há dissimilaridade em relação à avaliação das cascas da maioria das variedades avaliadas, com exceção da Niágara. De acordo com trabalho desenvolvido por Souza et al. (2012), estudando as mesmas variedades estudadas no presente trabalho, verificou-se que, para a maioria das análises físico-químicas, bromatológicas e de minerais realizadas, as maiores quantidades geralmente foram verificadas nas cascas das uvas. Yilmaz et al. (2015), estudando 22 variedades de uva em duas safras distintas, verificaram que o conteúdo de compostos fenólicos e a atividade antioxidante das polpas das uvas estudadas eram menores que o verificado nas sementes e nas cascas, respectivamente. Nesse mesmo trabalho, os autores verificaram que as uvas tintas apresentaram,

Tabela 1. Análise de correlação de compostos fenólicos, polifenoloxidase, peroxidase, índice de atividade antioxidante e ácido ascórbico, pelo Método de Pearson.

\begin{tabular}{|c|c|c|c|c|c|}
\hline Variáveis & $\begin{array}{l}\text { Compostos } \\
\text { Fenólicos }\end{array}$ & $\begin{array}{l}\text { Polifenol } \\
\text { Oxidase }\end{array}$ & Peroxidase & $\begin{array}{c}\text { Índice de Atividade } \\
\text { Antioxidante }\end{array}$ & $\begin{array}{c}\text { Ácido } \\
\text { Ascórbico }\end{array}$ \\
\hline Compostos Fenólicos & 1 & & & & \\
\hline Polifenol Oxidase & $0,44^{\star}$ & 1 & & & \\
\hline Peroxidase & $-0,05$ & $-0,27$ & 1 & & \\
\hline Índice de Atividade Antioxidante & 0,28 & 0,08 & $-0,25$ & 1 & \\
\hline Ácido Ascórbico & $0,51^{\star}$ & $0,80^{\star}$ & $-0,24$ & 0,11 & 1 \\
\hline
\end{tabular}

*Valores de correlação significativos para $\alpha=5 \%$. 


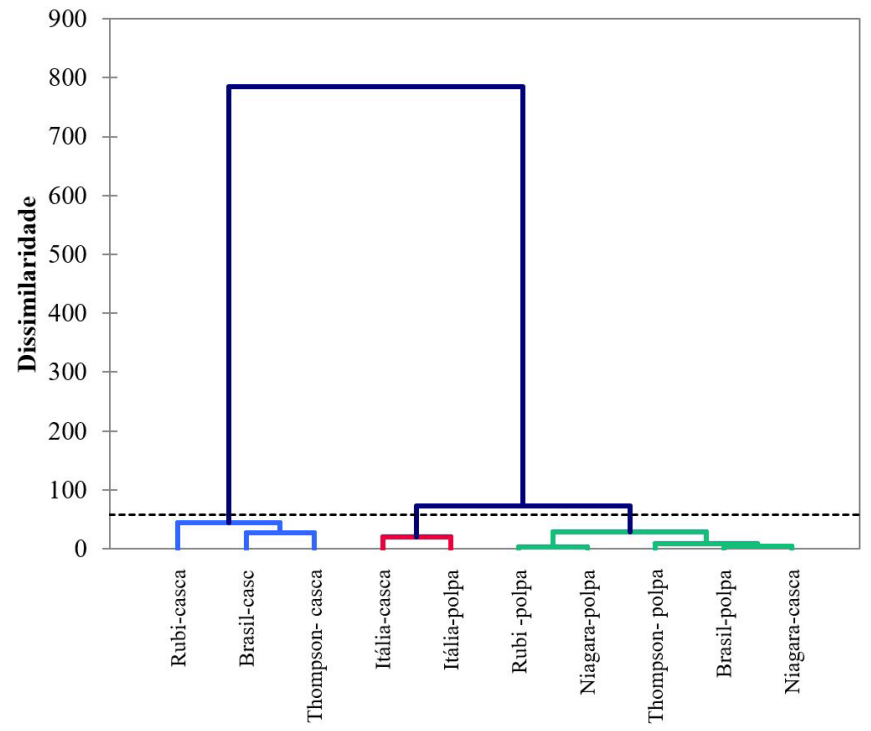

Figura 1. Dendrograma proveniente da análise de agrupamento para classificação das cultivares de uva de mesa.

em sua maioria, maiores teores de compostos fenólicos e atividade antioxidante que as variedades brancas, fato que é devido ao teor de antocianinas presentes nas variedades tintas. Resultados semelhantes foram encontrados no presente estudo, com exceção da variedade Thompson Seedless, que é uma uva branca. A quantidade de compostos fenólicos extraíveis para fabricação de vinhos é de cerca de $10 \%$ provenientes das polpas, enquanto $60 \%$ são representados pelas sementes e $30 \%$ pelas cascas (KARA et al., 2003).

A visível aproximação da maioria das cascas e das polpas entre si evidencia que as quantidades dos componentes analisados e as atividades enzimáticas foram discrepantes quando comparadas a esses parâmetros das partes dos frutos.

A análise multivariada foi realizada para verificar o agrupamento das diferentes respostas e obter informações adicionais acerca da influência das variáveis analisadas em relação às amostras de uva analisadas (Figura 2). Verificou-se que os componentes 1 e 2 (variedades e partes do fruto, e avaliações, respectivamente) explicaram $75,01 \%$ da variância do experimento. Contudo, pode-se verificar que existe estreita aproximação em relação aos compostos fenólicos, à atividade da enzima polifenoloxidase, ao teor de ácido ascórbico e à avaliação da casca da maioria das variedades. Dados estes que corroboram com Souza et al. (2010), que, em seus estudos, verificaram que as cascas das uvas avaliadas apresentaram maiores quantidades de ácido ascórbico para todas as mesmas variedades estudadas. Já com relação à maioria das polpas, verificou-se que elas apresentaram valores que se distanciam das análises realizadas, mostrando-se, portanto, mais pobres nas quantidades destes compostos

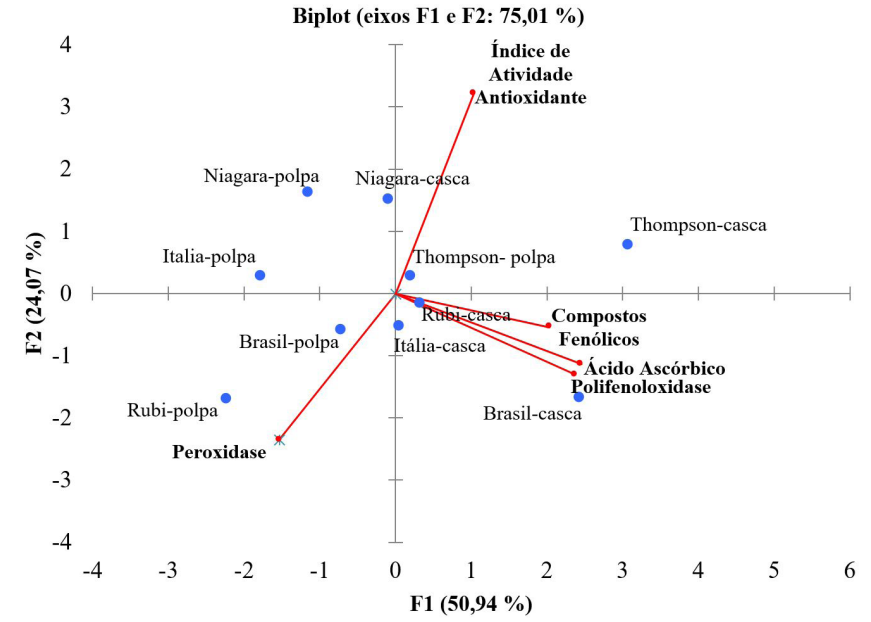

Figura 2. Análise de componente principal da atividade antioxidante em polpa e casca de uva de mesa de determinadas cultivares.

e atividades. Santos et al. (2011), estudando diferentes partes (casca, polpa e sementes) de quatro variedades de uva - Brasil (V. vinifera), Benitaka (V. vinifera), Isabel (Vitis labrusca) e Niágara (V. labrusca) -, verificaram que as sementes tiveram maior teor de compostos fenólicos, seguidas pela casca e polpa.

\section{Conclusão}

O estudo mostrou que, no geral, as cascas das uvas se correlacionam positivamente com o conteúdo de compostos fenólicos, a atividade da enzima polifenoloxidase e com a quantidade de ácido ascórbico. As cascas se mostraram uma ótima fonte para estes compostos e, assim, não devem ser desconsideradas na alimentação humana. Não houve correlação entre o índice de atividade antioxidante com as demais análises realizadas.

\section{Referências}

ASADI, S.; AHMADIANI, A.; ESMAEILI, M. A.; SONBOLI, A.; ANSARI, N.; KHODAGHOLI, F. In vitro antioxidant activities and an investigation of neuroprotection by six Salvia species from Iran: a comparative study. Food and Chemical Toxicology, v. 48, n. 5, p. 1341-1349, 2010. PMid:20197079. http://dx.doi. org/10.1016/j.fct.2010.02.035.

AWAD, M. Fisiologia pós-colheita de frutos. São Paulo: Nobel, 1993. $114 \mathrm{p}$.

BAGCHI, D.; BAGCHI, M.; STOHS, S. J.; DAS, D. K.; RAY, S. D.; KUSZYNSKI, C. A.; JOSHI, S. S.; PRUESS, H. G. Free radicals and grape seed proanthocyanidin extract: importance in human health and disease prevention. Toxicology, v. 148, n. 2-3, p. 187-197, 2000. PMid:10962138. http://dx.doi.org/10.1016/S0300483X(00)00210-9

BURIN, V. M.; FERREIRA-LIMA, N. E.; PANCERI, C. P.; BORDIGNONLUIZ, M. T. Bioactive compounds and antioxidant activity of 
Vitis vinifera and Vitis labrusca grapes: evaluation of different extraction methods. Microchemical Journal, v. 114, p. 155-163, 2014. http://dx.doi.org/10.1016/j.microc.2013.12.014

CANO, M. P.; ANCOS, O.; MATALLANA, M.; CÁMARA, M.; REGLERO, G.; TABERA, J. Differences among Spanish and Latin-American banana cultivars: morphological, chemical and sensory characteristics. Food Chemistry, v. 59, n. 3, p. 411-419, 1997. http://dx.doi.org/10.1016/S0308-8146(96)00285-3.

CETIN-KARACA, H.; NEWMAN, M. Antimicrobial efficacy of plant phenolic compounds against Salmonella and Escherichia coli. Food Bioscience, v. 11, p. 8-16, 2015. http://dx.doi.org/10.1016/j. fbio.2015.03.002.

CRUZ, C. D.; REGAZZI, A. J.; CARNEIRO, P. C. S. Divergência genética. In: CRUZ, C. D.; REGAZZI, A. J.; CARNEIRO, P. C. $S$. (Ed.). Modelos biométricos aplicados ao melhoramento genético. Viçosa: UFV, 2004.

DUMITRIU, D.; PEINADO, R. A.; PEINADO, J.; LERMA, N. Grape pomace extract improves the in vitro and in vivo antioxidant properties of wines from sin light dried Pedro Ximénez grapes. Journal of Functional Foods, v. 17, p. 380-387, 2015. http:// dx.doi.org/10.1016/j.jff.2015.06.003.

GONÇALVES, G. A.; SOARES, A. A.; CORREA, R. C. G.; BARROS, L.; HAMINIUK, C.; PERALTA, R. M.; FERREIRA, I. C. F. R.; BRACHT, A. Merlot grape pomace hydroalcoholic extract improves the oxidative and inflammatory states of rats with adjuvant-induced arthritis. Journal of Functional Foods, v. 33, p. 408-418, 2017. http://dx.doi.org/10.1016/j.jff.2017.04.009.

GONDIM, J. A. M.; MOURA, M. F. V.; DANTAS, A.; MEDEIROS, R. L. S.; SANTOS, K. M. Composição centesimal e de minerais em cascas de frutas. Ciência e Tecnologia de Alimentos, v. 25, n. 4, p. 825-827, 2005. http://dx.doi.org/10.1590/S010120612005000400032.

GRIS, E. F.; MATTIVI, F.; FERREIRA, E. A.; VRHOVSEK, U.; WILHELM FILHO, D.; PEDROSA, R. C.; BORDIGNON-LUIZ, M. T. Stilbenes and tyrosol as target compounds in the assessment of antioxidant and hypolipidemic activity of Vitis vinifera red wines from southern Brazil. Journal of Agricultural and Food Chemistry, v. 59, n. 14, p. 7954-7961, 2011. PMid:21718033. http://dx.doi.org/10.1021/jf2008056.

GUO, C.; YANG, J.; WEI, J.; LI, Y.; XU, J.; JIANG, Y. Antioxidant activities of peel, pulp and seed fractions of common fruits as determined by FRAP assay. Nutrition Research, v. 23, n. 12, p. 1719-1726, 2003. http://dx.doi.org/10.1016/j.nutres.2003.08.005.

JARA-PALACIOS, M. J.; HERNANZ, D.; CIFUENTES-GOMEZ, T.; ESCUDERO-GILETE, M. L.; HEREDIA, F. J.; SPENCER, J. P. Assessment of white grape pomace from winemaking as source of bioactive compounds, and its antiproliferative activity. Food Chemistry, v. 183, p. 78-82, 2015. PMid:25863613. http://dx.doi. org/10.1016/j.foodchem.2015.03.022.
KARA, F.; BOZ, Y.; UYSAL, T. The change of phenolic materials at the technological aspect of some black wine grape varieties under tekirdağ conditions. Turkey: Viticulture Research Institute; Ministry of Agriculture and Rural Affairs; General Directorate of Agricultural Research, 2003.

LAJILI, S.; AZOUAOU, S. A.; TURKI, M.; MULLER, C. D.; BOURAOUI, A. Anti-inflammatory, analgesic activities and gastro-protective effects of the phenolic contents of the red alga, Laurencia obtusa. European Journal of Integrative Medicine, v. 8, n. 3, p. 298-306, 2016. http://dx.doi.org/10.1016/j.eujim.2015.12.006.

LEE, S. Y.; MUNEROL, B.; POLLARD, S.; YOUDIM, K. A.; PANNALA, A. S.; KUHNLE, G. G.; DEBNAM, E. S.; RICE-EVANS, C.; SPENCER, $J$. P. The reaction of flavonols with nitrous acid protects against $\mathrm{N}$-nitrosamine formation and leads to the formation of nitroso derivatives which inhibit cancer cell growth. Free Radical Biology \& Medicine, v. 40, n. 2, p. 323-334, 2006. PMid:16413414. http:// dx.doi.org/10.1016/j.freeradbiomed.2005.08.031.

LIMA, G. P. P.; BRASIL, O. G.; OLIVEIRA, A. M. Poliaminas e atividade da peroxidase em feijão (Phaseolus vulgaris L.) cultivado sob estresse salino. Scientia Agrícola, v. 56, n. 1, p. 21-26, 1999. http://dx.doi.org/10.1590/S0103-90161999000100004.

LÓPEZ-MIRANDA, S.; SERRANO-MARTÍNEZ, A.; HERNÁNDEZSÁNCHEZ, P.; GUARDIOLA, L.; PÉREZ-SÁNCHEZ, H.; FORTEA, I.; GABALDÓN, J. A.; NÚÑEZ-DELICADO, E. Use of cyclodextrins to recover catechin and epicatechin from red grape pomace. Food Chemistry, v. 203, p. 379-385, 2016. PMid:26948628. http://dx.doi.org/10.1016/j.foodchem.2016.02.100.

MANACH, C.; WILLIAMSON, G.; MORAND, C.; SCALBERT, A.; RÉMÉSY, C. Bioavailability and bioefficacy of polyphenols in humans. I. Review of 97 bioavailability studies. The American Journal of Clinical Nutrition, v. 81, n. 1, p. 230S-242S, 2005. Supplement.

NIXDORF, S. L.; HERMOSÍN-GUTIÉRREZ, I. Brazilian red wines made from the hybrid grape cultivar Isabel: phenolic composition and antioxidant capacity. Analytica Chimica Acta, v. 659, n. 1-2, p. 208-215, 2010. PMid:20103126. http://dx.doi.org/10.1016/j. aca.2009.11.058.

SANTOS, L. P.; MORAIS, D. R.; SOUZA, N. E.; COTTICA, S. M.; BOROSKI, M.; VISENTAINER, J. V. Compostos fenólicos e ácidos gordos em diferentes partes de Vitis labrusca e V. vinifera. Food Research International, v. 44, n. 5, p. 394-400, 2011. http:// dx.doi.org/10.1016/j.foodres.2011.02.022.

SCHERER, R.; GODOY, H. T. Antioxidant activity index (AAI) by the 2,2-diphenyl-1-picrylhydrazyl method. Food Chemistry, v. 112, n. 3, p. 654-658, 2009. http://dx.doi.org/10.1016/j. foodchem.2008.06.026.

SINGLETON, V. L.; ROSSI JÚNIOR, J. A. Colorimetry of total phenolics with phosphomolybidic-phosphotungstic acid reagents. American Journal of Enology and Viticulture, v. 16, p. 144-158, 1965. 
SOARES, A. D.; GOMEZ, M. L. P. A.; MESQUITA, C.; LAJOLO, F. M. Ascorbic acid biosyntesis: a precursor study on plants. Brazilian Journal of Plant Physiology, v. 16, n. 3, p. 147-154, 2004. http://dx.doi.org/10.1590/S1677-04202004000300004.

SOUZA, A. V.; GOMES, G. P.; VIEIRA, M. R. S.; VIEITES, R. L.; LIMA, G. P. P. Atividade antioxidante em polpa de Vitis sp.

Revista Alimentus, v. 2, n. 2, p. 10-19, 2012.

SOUZA, A. V.; LIMA, G. P. P.; VIEITES, R. L. Avaliação nutricional de diferentes variedades de uva (Vitis sp). Naturalia, v. 33, p. 100-109, 2010.

TAGLIAZUCCHI, D.; VERZELLONI, E.; BERTOLINI, D.; CONTE, A. In vitro bio-accessibility and antioxidant activity of grape polyphenols. Food Chemistry, v. 120, n. 2, p. 599-606, 2010. http://dx.doi.org/10.1016/j.foodchem.2009.10.030.

TERADA, M.; WATANABE, Y.; KUNITOMA, M.; HAYASHI, E. Differential rapid analysis ascorbic acid and ascorbic acid 2-sulfate by dinitrophenilhydrazine method. Annals of Biochemistry, v. 4, n. 2, p. 604-608, 1979.

VÍTOLO, H. F.; SOUZA, G. M.; SILVEIRA, J. Cross-scale multivariate analysis of physiological responses to high temperature in two tropical crops with C3 and C4 metabolism. Environmental and Experimental Botany, v. 80, n. 1, 2012.

VU, K. D.; CARLETTINI, H.; BOUVET, J.; CÔTÉ, J.; DOYON, G.; SYLVAIN, J.; LACROIX, M. Effect of different cranberry extracts and juices during cranberry juice processing on the antiproliferative activity against two colon cancer cell lines. Food Chemistry, v. 132, n. 2, p. 959-967, 2012. http://dx.doi. org/10.1016/j.foodchem.2011.11.078.

YILMAZ, Y.; GÖKSEL, Z.; ERDOĞAN, S. S.; ÖZTÜRK, A.; ATAK, A.; ÖZER, C. Antioxidant activity and phenolic content of seed, skin and pulp parts of 22 grape (Vitis vinifera L.) cultivars (4 common and 18 registered or candidate for registration). Journal of Food Processing and Preservation, v. 39, n. 6, p. 1682-1691, 2015. http://dx.doi.org/10.1111/jfpp.12399. 